\title{
Shared lexical-semantic features and the animacy effect in early-course psychosis
}

\author{
Martina Sekulić Sović ${ }^{1}$ Vlasta Erdeljac ${ }^{1}$ Iva Kužina ${ }^{1}$ Mija Vandek ${ }^{1}$ \\ - Ninoslav Mimica ${ }^{2,3}$ • Draženka Ostojićz ${ }^{2,4}$ - Aleksandar Savićź, \\ 'University of Zagreb, Faculty of Humanities and Social Sciences, Department of Linguistics, \\ ${ }^{2}$ University Psychiatric Hospital Vrapče, ${ }^{3}$ University of Zagreb, School of Medicine, Chair of \\ Psychiatry and Psychological Medicine, and ${ }^{4}$ University of Zagreb, Faculty of Law, Social Work \\ Study Centre
}

https://doi.org/10.17234/9789531758314.06

\begin{abstract}
Language deficits in psychosis, and in schizophrenia, are presumed to be due to increased activation and connectivity of the semantic memory, which is determined by lexical-semantic features of concepts. The aim of this study was to analyse the influence of shared lexical-semantic features on language processing in patients with first-episode and early-course psychosis. The study included 15 Croatian-speaking patients from the University Psychiatric Hospital Vrapče, Zagreb, diagnosed with first-episode and early-course psychosis and a healthy control group. The subjects performed a lexical-semantic decision task in which the primes and the target words were either related as hypernym and hyponym or were unrelated, and in which the target words represented either animate or inanimate concepts. Two results analyses were conducted: one on the taxonomic (hyponym-hypernym) condition and one on the animacy (living/non-living stimulus) condition. The patient group was less accurate on the taxonomy condition because the taxonomy relations were dependent on their shared features. The patients' activation of shared features was higher, and their inhibition was reduced. Consequently, the patients will have a greater number of concepts activated and not inhibited. For the inanimate concepts, a high correlation of distinctive features is characteristic, while the animate concepts have a high correlation of shared features. The presupposition is that the greater activation of shared features influenced the patients' answers, so the distinctive features had no influence. Thus, the control group had higher accuracy for inanimate concepts.
\end{abstract}

Key words: lexical-semantic features, early-onset psychosis, taxonomy relations, animacy 


\section{Introduction}

The language dysfunction in schizophrenia is explained with a theory that attributes language abnormalities to abnormalities in the structure and function of semantic memory, which in turn lead to the language abnormalities (Spitzer et al., 1993; Aloia et al., 1998, as cited in Kuperberg, 2010). Perhaps the most influential theory of positive thought disorder in schizophrenia is connected with the hyperactivity of spreading activation in semantic memory (Ballerini, 2016) which stems from faster and more automatic spread of activation through semantic memory (Manschreck et al., 1988; Spitzer et al., 1993, as cited in Kuperbeg, 2010). This model is tied to the network model of semantic memory (Collins \& Loftus, 1975), which presupposes its organization as a structure of concepts in a distributed connectionist system memory in which words and concepts are linked within a network according to their degree of association or co-occurrence (Collins \& Loftus, 1975; Anderson, 1983, as cited in Kuperberg, 2010). Each concept is represented by a node of interconnected links which are different overlapping features and relations of the concept. The activation of concepts in this model depends on the activation and overlapping of feature activation and the connectivity of the network (Minzerberg, 2002; Tyler \& Moss, 2001), which is presumed to be increased in schizophrenia. A higher activation of concepts in the semantic memory requires a heightened inhibition of the concepts which have been activated, and this inhibition is believed to decrease in psychosis and schizophrenia. Furthermore, in psychotic disorders, and specifically in schizophrenia, cognitive and lexical-semantic deficits may occur as a syndrome of dysfunctional connectivity and functional changes in the language network (Agcaoglu et al., 2017; Cavelti et al., 2018).

Furthermore, semantic deficits associated with FTD (formal thought disorder) could include impaired access to semantic concepts (Leeson et al., 2005), as well as degraded or disorganized storage of semantic information (Rossell \& David, 2006, as cited in Summer et al., 2018). Assaf et al. (2007) suggest that FTD symptoms in schizophrenia may not extend from a disruption of the semantic network but are secondary to a selective dysfunction in specific components of semantic operations related to semantic object retrieval. They showed that FTD is specifically associated with impaired object recall from features in the semantic memory and not with other semantic processes (such as synonym and category judgment). Furthermore, they demonstrated the neural abnormalities that underlie this semantic deficit. The patients retrieved more objects based on unrelated (distant) features and did so more slowly than the healthy controls. These results are generally in accord with the hypothesis of far-spreading activation.

From a psycholinguistic point of view, word retrieval depends on the connectivity of the language network and can be facilitated by shared lexical-seman- 
tic features. A question arises concerning the effect of different types of shared and distinctive features. According to the conceptual structure account (Tyler \& Moss, 2001), concepts that share the animacy feature predict more correlated intercategory features (and weak correlation of distinctive properties), while inanimate concepts have higher correlation of distinctive features (and weak correlation of shared properties). The animacy feature refers both to the quality and quantity of features. Greater correlations in shared features facilitate the activation of a greater number of conceptual features of the same category, suggesting that the activation of animate concepts will co-activate a more distributed conceptual network. In a study on a speeded feature verification task carried out on healthy participants, Randall et al. (2004) concluded that the subjects processed distinctive, but highly correlated, features of inanimate concepts faster than distinctive features of inanimate concepts that are not highly correlated, but Cree et al. (2006) found no differences in the processing speed of distinctive features of animate and inanimate concepts and concluded that they are both processed more quickly than shared features. Studies on pathologies with structural lesions stress the importance of distinctive features and predict the preservation of inanimate concepts on account of the preservation of highly correlated distinctive features. Since the distinction between animate and inanimate concepts in selective deficits in patients is common (Mahon \& Caramazza, 2003), Bonin et al. (2013) suggest an animacy effect category which refers to the presupposition that the stimuli with the animacy feature are processed differently than the stimuli without it (Van Arsadal et al., 2013, as cited in Bonin et al., 2013). A higher activation of shared features is expected in neurofunctional disorders such as first-episode and early-course psychosis, which results in greater activation of concepts in the semantic memory. We presuppose that more intercategory correlated features will account for an equal activation of a higher number of concepts in the same category. Correlated properties are those which frequently occur together; they are more robust to damage because they support one another with mutual activation (Pilgrima et al., 2013; Tyler \& Moss, 2001).

Furthermore, taxonomy relations are also dependent on their shared features. From a neurocognitive view, taxonomic categories are generally similarity-based; that is, they have shared attributes, while thematic categories are not based on similarity (i.e. shared features) but on extrinsic relations between two objects. Taxonomic knowledge tells us the properties of a set of objects, whereas thematic knowledge tells us how other categories relate to that set (Murphy, 2010, as cited in Lewis et al., 2015). While taxonomic categories are represented only by their associations to features (Rogers \& McClelland, 2004), they do not include relations formed by thematic knowledge. Nevertheless, while taxonomic knowledge includes the properties of an object and its concept, thematic knowledge also includes taxonomic knowledge, as it needs to show how other 
categories and their constituents relate to the features of concepts so it can form a thematic relation. As taxonomic categories are similarity based and fall under the same superordinate relations, their features are largely shared. When concepts are activated, their relatedness will differ in terms of the amount of information linking them. The higher the relatedness effect, the faster the subjects will answer, due to saliency and the number of their relations. Since taxonomy categories presuppose a high number of shared features, their relatedness effect is expected to be highly accounted for. In a neurofunctional imaging study, Lewis et al. (2015) raised the question of whether taxonomic and thematic information are represented together, and their results show that taxonomic relations strongly predicted anterior temporal lobe activation, and both kinds of relations influenced the temporoparietal junction.

According to the theoretical framework that describes schizophrenia as a syndrome of an inadequate integration of connections and global functional changes in brain regions that are crucial for language processing, the presupposition is that highly correlated shared features will be activated more quickly and that the activation will spread faster within them in the semantic memory.

Greater correlations in intercategory features facilitate the activation of a higher number of conceptual features of the same semantic category, suggesting that the activation of animate concepts co-activates a more distributed conceptual network. The assumption is that patients with early-course psychosis will have greater activation in the semantic memory for animate words than for inanimate ones in hierarchy relations because of the stronger correlation of shared properties.

Moreover, it is assumed that there will be no differences in accuracy with inanimate concepts between the two subject groups, while the patients will have lower accuracy on animate concepts because of a higher activation of shared features. Higher activation of shared features is also expected with the taxonomy condition. Since subjects with first-episode and early-course psychosis have reduced inhibition, which is necessary on both conditions as there is higher activation, the assumption is that the patients will have a higher amount of incorrect answers because relations and concepts will be activated and not inhibited.

The aim of this study was to examine how the activation of shared features influences lexical-semantic processing in patients with first-episode and early-course psychosis. The patients were expected to be significantly statistically less accurate on taxonomy relations (hyponymy and hyperonymy condition) on account of the activation of shared features. Furthermore, the control group was expected to have statistically higher accuracy on inanimate concepts because of the activation of distinctive features, while the patient group results on accuracy would not differ on animate and inanimate concepts because of a heightened activation of shared features. 


\section{Methods}

\subsection{Participants}

The study recruited 15 Croatian-speaking patients from the University Psychiatric Hospital Vrapče, Zagreb, diagnosed with first-episode and early-course psychosis. On average, the patients were 26.85 years old and had finished 13.6 years of education. At the time of the study, their average time after the onset of illness was 9.07 months. Their average time after the initiation of therapy was 5.13 months. All patients were receiving antipsychotic treatment, and their average daily dose of antipsychotics expressed in chlorpromazine equivalents was $507.78 \mathrm{mg}$. Informed consent had been obtained from all of the participants before the administration of the test, and the test had been approved by the Ethics Committee of the University Psychiatric Hospital Vrapče (Registry number: 23-305/8-18). The control group for the first analysis consisted of 15 healthy subjects, and for the second, 19 healthy subjects, matched with the patients by age, sex, and by their dominant hand.

\subsection{Materials and procedure}

The subjects performed a lexical-semantic decision task. The stimuli were presented visually on a computer screen. In some cases, the prime and the target word were related as hyponym and hypernym, while in others, they were unrelated. In addition, the target words represented either animate or inanimate concepts. The prime word was presented for $1000 \mathrm{~ms}$. A 100-ms window followed, after which the target word was presented for $3000 \mathrm{~ms}$. The stimuli were used and consistently balanced, having been taken from Psiholeks_HR (Erdeljac, Sekulić Sović \& Miklić, 2018), an online psycholinguistic database including measures for 2000 Croatian words based on data collected from 100 participants. In the database, each word is described according to specified linguistic criteria (morphosyntactic: part of speech; phonological-phonetic-orthographic: number of syllables, number of phonemes, number of letters), and each word is attributed with the measures and values of five psycholinguistic parameters: subjective word frequency, imageability, abstractness/concreteness, word familiarity, age of acquisition, and word associations. The subjects answered yes or no to the following question: "Does the second word you read represent an animate concept?". Accuracy and reaction times were measured using E-prime 2.0 software (Psychology Software Tools, Pittsburgh) (Schneider et al., 2012). Two independent analyses were conducted, each depending on the different organization of the stimulus materials. The first analysis included 30 stimulus word-pairs, 15 word-pairs in a hyponymy/hypernymy relation and 15 not related in any taxonomy relation. The second analysis included 26 stimulus word-pairs, 13 with the animacy feature (i.e. representing living concepts) and 13 without the animacy feature (i.e. representing non-living concepts). 


\section{Results}

\subsection{Statistical analysis of the taxonomy condition}

The data distribution was non-normal, and the variances were not homogeneous. Therefore, non-parametric analyses were applied. Differences in the accuracy and time reaction between the two conditions (lexical-semantically related vs. unrelated words) were analysed using a Wilcoxon signed-rank test and Friedman ANOVA, respectively, while Whitney $U$ test was used for testing differences between two independent groups. $P$ value was set to .05 ; however, Bonferroni correction for multiple comparisons was applied. Statistical analyses were performed using IBM SPSS Statistics 23.0.

Table 1. Descriptive data (median and interquartile range) for the accuracy and reaction time on lexical-semantically related and unrelated words in the control and patient groups

\begin{tabular}{|l|r|r|r|r|r|r|}
\hline & \multicolumn{3}{|c|}{$\begin{array}{c}\text { Controls } \\
(N=15)\end{array}$} & \multicolumn{3}{c|}{$\begin{array}{c}\text { Patients } \\
\text { (N= 15) }\end{array}$} \\
\hline & Mdn & \multicolumn{1}{|c|}{ Q1 } & \multicolumn{1}{c|}{ Q3 } & \multicolumn{1}{c|}{ Mdn } & Q1 & \multicolumn{1}{|c|}{ Q3 } \\
\hline Accuracy & & & & & & \\
\hline Related words & 93.33 & 93.33 & 100.00 & 93.33 & 80.00 & 93.33 \\
\hline Unrelated words & 100.00 & 100.00 & 100.00 & 100.00 & 73.33 & 100.00 \\
\hline Accuracy (\%) & & & & & & 12 \\
\hline Related words & 14 & 14 & 15 & 14 & 11 & 14 \\
\hline Unrelated words & 15 & 15 & 15 & 15 & 11 & 14 \\
\hline Reaction time (ms) & & & & & & 1395.71 \\
\hline Related words & 704.20 & 634.09 & 765.50 & 978.30 & 847.80 & 1437.64 \\
\hline Unrelated words & 693.00 & 608.73 & 760.33 & 1029.54 & 808.73 & 15 \\
\hline
\end{tabular}

The Wilcoxon signed-rank test for dependent samples showed that there was no significant difference in accuracy between related and unrelated words in the control group $(T(15)=7.0, z=1.18, p=0.2367, r=.31)$, nor in the patients' group $(T(15)=34.00, z=0.39, p=.6949, r=.10)$, which can be seen in Figure 1. However, there was a significant difference in accuracy between the groups: a lower percentage of accuracy was observed in the patients than in the controls, but only for related words $(U(15,15)=62.00, z=2.27, p=.0229, r=.41)$, while there was no difference in accuracy between the two groups for the unrelated words $(U(15,15)=77.50, z=1.73, p=.0834, r=.32)$. 


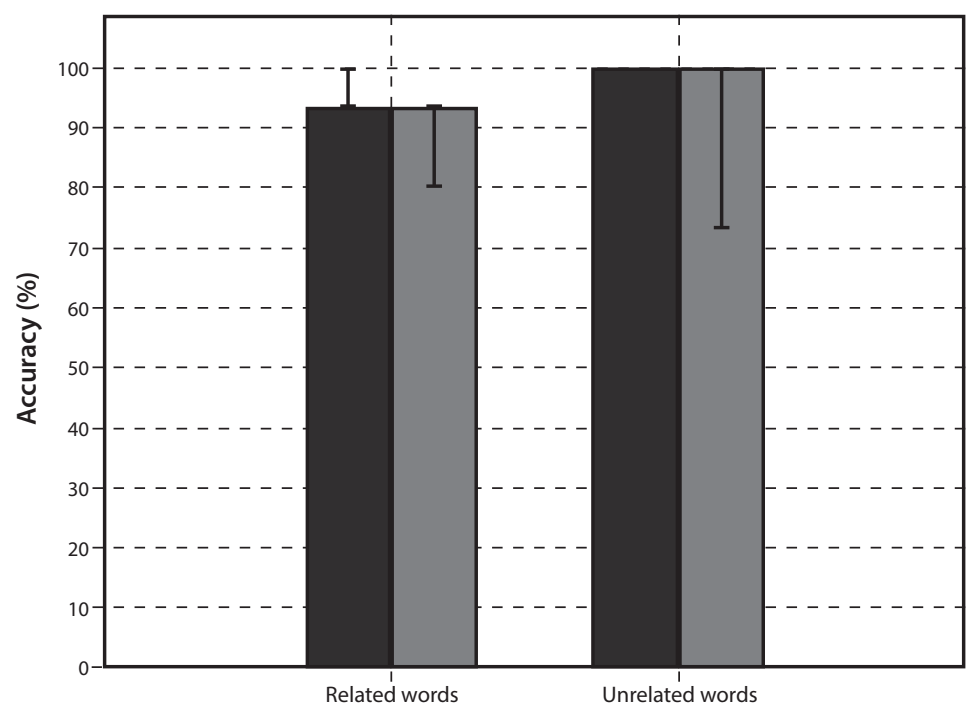

Figure 1. Control- and patient-group accuracy for related and unrelated words (median and interquartile range presented)

There was no significant difference in the reaction time (ms) between related and unrelated words either in the control group $(T(15)=43.00, z=0.97, p=$ $.3343, r=.25)$ or in the patients' group $(T(15)=41.00, z=1.08, p=.2805, r=.28)$, which can be seen in Figure 2. However, there was a significant difference in the reaction time between the groups, where longer reaction times were observed in the patients than in the controls both for related words $(U(15,15)=8.00, z$ $=-4.33, p<0.001, r=-.79)$ and unrelated words $(U(15,15)=21.00, z=-3.80$, $p<0.001, r=-.69)$. 


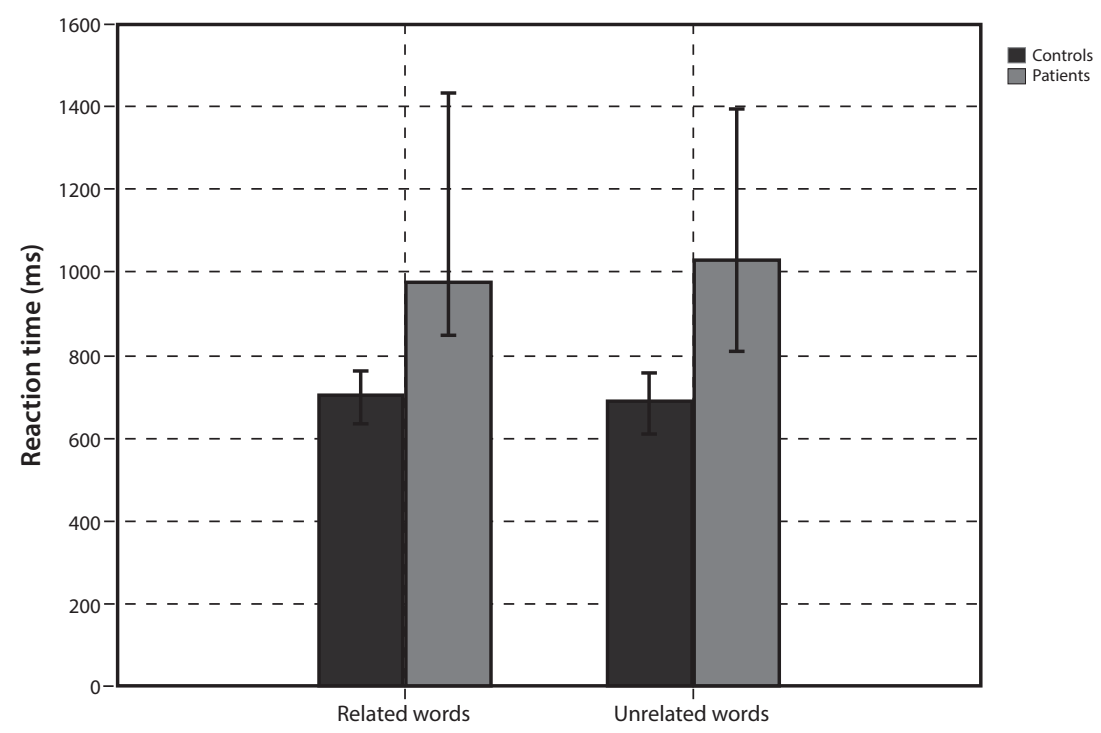

Figure 2. Reaction time (ms) of control and patient groups for related and unrelated words (median and interquartile range presented)

\subsection{Statistical analysis of the animacy condition}

The data distribution was non-normal, and the variances were not homogeneous. Therefore, non-parametric analyses were applied. The differences in accuracy and reaction time between the two conditions (animate vs. inanimate) were analysed using a Wilcoxon signed-rank test and Friedman's ANOVA, respectively, while a Whitney $\mathrm{U}$ test was used to test the differences between the two independent groups. The p-value was set to .05; however, the Bonferroni correction for multiple comparisons was applied. Statistical analyses were performed using IBM SPSS Statistics 23.0.

Table 2. Descriptive data (median and interquartile range) on the accuracy and reaction time of the control and patient groups for animate and inanimate words

\begin{tabular}{|l|r|r|r|r|r|r|}
\hline & \multicolumn{3}{|c|}{ Controls (N= 19) } & \multicolumn{3}{c|}{ Patients (N= 15) } \\
\hline & \multicolumn{1}{|c|}{ Mdn } & \multicolumn{1}{c|}{ Q1 } & \multicolumn{1}{c|}{ Q3 } & \multicolumn{1}{c|}{ Mdn } & \multicolumn{1}{c|}{ Q1 } & \multicolumn{1}{c|}{ Q3 } \\
\hline Accuracy & & & & & & \\
\hline Animate words & 13.00 & 12.00 & 13.00 & 12.00 & 10.00 & 13.00 \\
\hline Inanimate words & 13.00 & 13.00 & 13.00 & 13.00 & 9.00 & 13.00 \\
\hline Accuracy (\%) & & & & & & \\
\hline Animate words & 100.00 & 92.31 & 100.00 & 92.31 & 76.92 & 100.00 \\
\hline Inanimate words & 100.00 & 100.00 & 100.00 & 100.00 & 69.23 & 100.00 \\
\hline Reaction time(ms) & & & & & & \\
\hline Animate words & 736.23 & 636.77 & 797.08 & 963.58 & 842.17 & 1278.77 \\
\hline Inanimate words & 653.54 & 614.64 & 712.85 & 1055.69 & 792.00 & 1394.25 \\
\hline
\end{tabular}


The Wilcoxon signed-rank test for dependent samples showed that the patients' group displayed no significant difference in accuracy for animate vs. inanimate words in $(T(15)=30.00, z=0.27, p=0.7897, r=.07)$, which can be seen in Figure 1 . However, the accuracy of the control group was significantly higher for the inanimate words than for animate words $(T(15)=0.00, z=2.02, p=.04312, r$ $=.52$ ). Although there was no significant difference between the groups for the animate words $(U(15,15)=71.00, z=1.85, p=0.0637, r=.34)$, there was a significant difference between the groups for the inanimate words $(U(15,15)=60.00$, $z=2.18, p=0.0033, r=.40)$, where the control group had a higher level of accuracy.

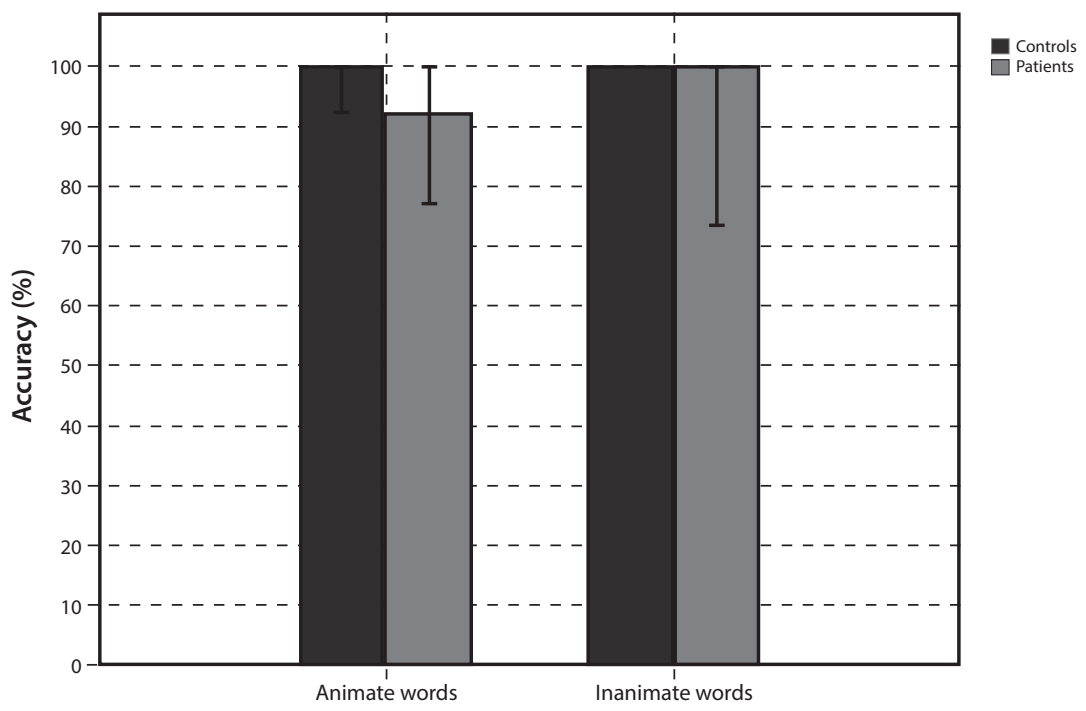

Figure 3. Control- and patient-group accuracy for animate and inanimate words (median and interquartile range presented)

When reaction time was taken into account, the Wilcoxon signed-rank test for dependent samples showed that there was no significant difference in the reaction time (ms) on animate and inanimate words among the control group $(T(15)$ $=26.00, z=1.93, p=0.0535, r=.50)$ nor among the patients' group $(T(15)=$ $53.00, z=0.40, p=0.6910, r=.10$ ), which can be seen in Figure 2. However, there was a significant difference in the reaction time between the two groups: longer reaction times were observed in the patients than in the controls for both animate words $(U(15,15)=14.00, z=-4.09, p<0.001, r=-.75)$ and inanimate words $(U(15,15)=19.00, z=-3.88, p<0.001, r=-.71)$. 


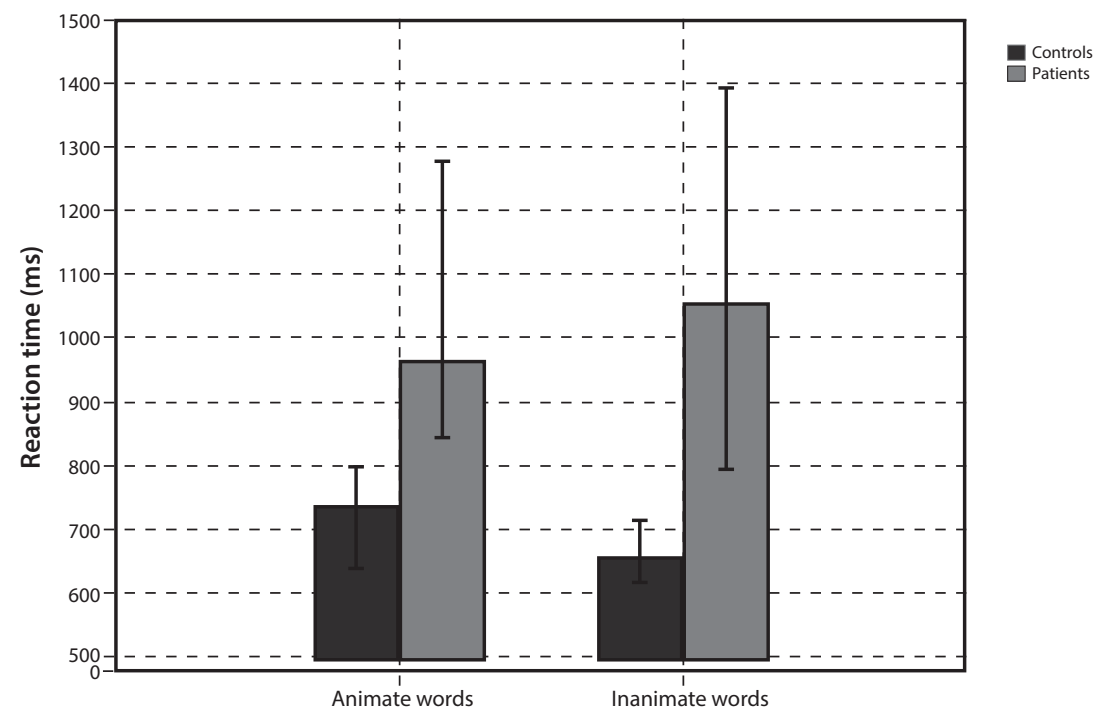

Figure 4. Control- and Patient-group reaction times (ms) on animate and inanimate words (median and interquartile range presented)

\section{Discussion}

The patient group was significantly less accurate on the taxonomy condition than the control group. The higher activation of shared features, both highly correlated shared features of animate concepts and weakly correlated shared features of inanimate concepts, demanded heightened inhibition, which failed and resulted in the activation of incorrect relations in the semantic memory and lower accuracy in the patient group. Furthermore, when shared features were not activated, there were no differences in accuracy between the two groups.

The control group had statistically higher accuracy on inanimate concepts, and the patient group's accuracy results did not differ on animate and inanimate concepts. Inanimate concepts have more distinctive features, both correlated and non-correlated, which influenced the answers of the control group the most. On the other hand, in the patient group there were no differences in accuracy, as shared features were highly activated in both the animate and the inanimate concepts.

According to Studerus et al. (2016), neurocognitive variables likely play an important role in multi-domain prediction models because cognitive deficits are considered core features of schizophrenic psychoses. The greatest impairments appear to be present in the domains of verbal memory, speed of processing, and working memory (e.g. Schaefer et al., 2013; Fatouros-Bergman et al., 2014; Mesholam-Gately et al., 2009). Furthermore, meta-analyses suggest that, at the onset of psychosis, cognitive performance is on average about one standard der- 
ivation (SD) below been consistently demonstrated in all cognitive domains with medium to large effect sizes and cannot be attributed to effects of antipsychotics because unmediated and medicated patients are similarly affected (e.g. Fatouros-Bergman et al., 2014). According to Riecher-Rössler and McGorry (2016), impaired performance across a wide range of cognitive domains, especially in the areas of working memory, verbal fluency verbal memory, and speed of information processing, could potentially be used for improving the accuracy of prediction (e.g. Riecher-Rössle et al., 2009; Fusar-Poli et al., 2012).

\section{Conclusion}

Assessment of the processing of specific lexical-semantic features alongside neuropsychological evaluation might be a valuable tool as an indicator and predictor of particular phases and/or courses of illness. Both analyses confirmed that the activation of shared features influenced language processing in subjects with first-episode and early-course psychosis differently than in healthy subjects. This influence can be observed in accordance with the model of spreading activation of the semantic memory in schizophrenia because the activation of shared features caused slower reaction times and a higher amount of incorrect answers in the patient group, which is presumed to be a result of greater activation and a reduced inhibition of the concepts in the semantic memory.

\section{References}

Agcaoglu, O., Miller, R., Damaraju, E., Rashid, B., Bustillo, J., Cetin, M. S., Van Erp, T. G. M., McEwen, S., Preda, A., Ford, J. M., Lim, K. O., Manoach, D. S., Mathalon, D. H., Potkin, S. G., \& Calhoun, V. D. (2018). Decreased hemispheric connectivity and decreased intra- and inter- hemisphere asymmetry of resting state functional network connectivity in schizophrenia. Brain Imaging and Behavior, 12(3), 615-630.

Assaf, M., Rivkin, P., Kraut, M., Calhoun, V., Hart, J. Jr, \& Pearlson, G. (2007). Applications of models to understanding cognitive dysfunction: Schizophrenia and semantic memory. In J. Hart, Jr. \& J. H. Kraut (Eds.), Neural Basis of Semantic Memory (pp. 133-145). Cambridge: Cambridge University Press.

Ballerini, M. (2016). Semantic processing and semantic experience in people with schizophrenia: A bridge between phenomenological psychopathology and neuroscience? Journal of Psychopathology, 22, 94-105.

Bonin, P., Gelin, M., \& Bugaiska, A. (2013). Animates are better remembered than inanimates: further evidence from word and picture stimuli. Memory \& Cognition, 42(3), 370-382.

Cavelti, M., Winkelbeiner, S., Federspiel, A., Walther, S., Stegmayer, K., Giezendanner, S., Laimböck, K., Dierks, T., Strik, W., Horn, H., \& Homan, P. (2018). Formal tho- 
ught disorder is related to aberrations in language-related white matter tracts in patients with schizophrenia. Psychiatry Research: Neuroimaging, 279, 40-50.

Collins, A. M. \& Loftus, A. S. (1975). A spreading activation theory of semantic processing. Psychological Review, 82, 407-428.

Cree, G. S., McNorgan, C., \& McRae, K. (2006). Distinctive features hold a privileged status in the computation of word meaning: Implications for theories of semantic memory. Journal of Experimental Psychology: Learning, Memory, and Cognition, 32, 643-658.

Erdeljac, V., Sekulić Sović, M., \& Miklić D. (2018). Psycholingustic Database - Psiholex_HR. Zagreb: Department of Linguistics, Faculty of Humanities and Social Sciences, University of Zagreb.

Fatouros-Bergman, H., Cervenka, S., Flyckt, L., Edman, G., \& Farde, L. (2014). Meta-analysis of cognitive performance in drug-naïve patients with schizophrenia. Schizophrenia Research, 158, 156-162.

Fusar-Poli, P., Deste, G., Smieskova, R., Barlati, S., Yung, A. R., Howes, O., Stieglitz, R. D., Vita, A., McGuire, P., \& Borgwardt, S. (2012). Cognitive functioning in prodromal psychosis: A meta-analysis. Archives of general psychiatry, 69(6), 562-571.

Kuperberg, G.R. (2010). Language in schizophrenia part 1: An introduction. Language and Linguistics Compass, 4, 576-589.

Leeson, V. C., Simpson, A., McKenna, P. J., \& Laws, K. R. (2005). Executive inhibition and semantic association in schizophrenia. Schizophrenia Research, 74, 61-67.

Lewis, G. A., Poeppel, D., \& Murphy, G. L. (2015). The neural bases of taxonomic and thematic conceptual relations: An MEG study. Neuropsychologia, 68, 176-189.

Mahon, B. Z., \& Caramazza, A. (2003). Constraining questions about the organisation and representation of conceptual knowledge. Cognitive Neuropsychology, 20, 433450.

Mesholam-Gately, R. I., Giuliano, A. J., Goff, K. P., Faraone, S. V., \& Seidman, L. J. (2009). Neurocognition in first-episode schizophrenia: A meta-analytic review. Neuropsychology, 23(3), 315-336.

Minzenberg, M. J., Ober, B. A., \& Vinogradov, S. (2002). Semantic priming in schizophrenia: A review and synthesis. Journal of the International Neuropsychological Society, 8(5), 699-720.

Pilgrima, L. K., Moss, H. E., \& Tyler, L. K. (2005). Semantic processing of living and nonliving concepts across the cerebral hemispheres. Brain and Language, 94, 86-93.

Randall, B., Moss, H. E., Rodd, J. M., Greer, M., \& Tyler, L. K. (2004). Distinctiveness and correlation in conceptual structure: Behavioral and computational studies. Journal of Experimental Psychology: Learning, Memory, and Cognition, 30, 393-406.

Riecher-Rössler A., \& McGorry P. D. (2016). Early detection and intervention in psychosis. In A. Riecher-Rössler \& P. D. McGorry (Eds.), Early Detection and Intervention in Psychosis: State of the Art and Future Perspectives (Key Issues in Mental Health, vol. 181, pp. 179-189) Basel: Karger.

Riecher-Rössler, A., Pflueger, M. O., Aston, J., Borgwardt, S. J., Brewer, W. J., Gschwandtner, U., \& Stieglitz, R. D. (2009). The efficacy of using cognitive status in predicting psychosis: A 7-year follow-up. Biological Psychiatry, 66(11), 1023-1030. 
Rogers, T. T., \& McClelland, J. L. (2004). Semantic cognition: A parallel distributed processing approach. Cambridge, MA: MIT Press.

Schaefer, J., Giangrande, E., Weinberger, D. R., \& Dickinson, D. (2013). The global cognitive impairment in schizophrenia: Consistent over decades and around the world. Schizophrenia Research, 150(1), 42-50.

Schneider, W., Eschman, A., \& Zuccolotto, A. (2012). Using E-prime, 2.0 software, Psychology Software Tools, Pittsburgh: Psychology Software Tools, Inc.

Studerus, E., Papmeyer, M., \& Riecher-Rössler, A. (2016). Neurocognition and motor functioning in the prediction of Psychosis. In A. Riecher-Rössler \& P. D. McGorry, (Eds.), Early detection and intervention in psychosis: State of the art and future perspectives (Key Issues in Mental Health, vol. 181, pp. 179-189). Basel: Karger.

Summer, P. J., Bell, I. H., \& Rossell, S. L. (2018). A systematic review of task-based functional neuroimaging studies investigating language, semantic and executive processes in thought disorder. Neuroscience and Biobehavioral Reviews, 94, 59-75.

Tyler, L. K., \& Moss, H. E. (2001). Towards a distributed account of conceptual knowledge. Trends in Cognitive Sciences, 5, 244-252. 